\title{
Los procesos de las narrativas Asháninca en la frontera entre Brasil y Perú
}

\section{Asháninca narratives and their processes on the border between Brazil and Peru}

\author{
Érika Mesquita \\ Universidade Estadual de Campinas \\ erikamesquita28@gmail.com
}

\section{Resumen}

En este artículo se discuten las narrativas de los ashánincas brasileños y cómo en estas están representadas algunas experiencias. Los mitos se mezclan con los procesos que los ashánincas están desarrollando para la comprensión de los fenómenos en su vida cotidiana. Centramos nuestra atención en el contexto de algunos de sus conocimientos; en particular, los que se refieren a su cosmología, la cual integra puntos de vista de muchos mundos, mezclando figuras fantásticas y apariciones sobrenaturales con la presentación de alguna representación de las versiones narrativas de su historia y los conocimientos adquiridos también a través de las plantas maestras en su hábitat: la región fronteriza del bosque entre Brasil y Perú.

Palabras clave: asháninca, etnografía, cosmología

\begin{abstract}
This article discusses the narratives and representations the some experiences that the tribe Asháninca that live in Brazil. The myths are complex and also the processes that the Asháninca are developing for the understanding of the phenomena in their daily life. We focus our attention on the context of some of his knowledge, particularly those referring to his cosmology, which integrates viewpoints of many worlds, mixing fantastic figures and supernatural appearances with the presentation of some representation of the narrated versions of Its history and the knowledge acquired also through the master plants in their habitat: the border region of the forest between Brazil and Peru.
\end{abstract}

Keywords: Ashaninca, Ethnography, Cosmology 


\section{Los Asháninca en tierras brasileñas}

Los Asháninca que viven actualmente en Acre son de Perú, de la región del Gran Pajonal, donde encontramos su origen. La población emigró en diferentes momentos a Brasil, debido a la presión del caucho. Algunos trabajaron para los patrones caucheros y otros en una incipiente industria de la madera que se inició en la frontera. Los que se hallaban hastiados de los excesos de los jefes caminaron por las cabeceras de la región del río Yurúa, región donde actualmente se encuentran las reservas Asháninca en Brasil ${ }^{1}$. Los Asháninca son hablantes de la familia lingüística Arawak de la región de Ucayali². Cazevitz Renard (1992) identifica el Aruak Asháninca o grupos Arawak, Nomatsigenga, Matsigenga y Amuesha $^{3}$. Los tres primeros grupos se conocen anteriormente como Campa o Kampa.

Esta población migró en diferentes épocas para lo Brasil por la presión del caucho, unos trabajando para los patrones del caucho y otros para patrones madereros peruanos; los que no estaban debajo de los mandos y desmanes de los patrones, deambulaban por las cabeceras del río Envira y de los ríos Breu y Amônia componentes de la región del alto río Juruá en tierras brasileñas. Todavía, hay una gran conexión de parentesco entre los Asháninca con los de la región de las cuencas de los ríos Ene, Tambo, Alto Perené, Pachitea, alto Ucayali, región que se encuentra el Gran Pajonal (Kishiatsi), con $1715 \mathrm{~km}$ aproximadamente de donde ese pueblo es natural. Según Marinki, Asháninca que recuerda de su papá hablando de su historia, habla que "en el río Tamaya, su territorio ancestral fue establecido en un tiempo mítico por diversas divinidades, como Avireri o Navireri, que hizo salir de su país de origen, el lago los virakotxa, existe el lago está en el distrito de Perené en la provincia de Chanchamayo, de donde vienen nuestros parientes". Los Asháninca más ancianos que viven en la aldea de la región de frontera llegaron a la región, venidos del Sheshea, donde se localiza el Amoninha, nombre dado al río Tamaya, en tierras brasileñas. Estos Ashánincas se fijaron en el trabajo de extracción de madera para empresas o patrones (watorote) peruanos. Esta región se constituye en un área pobre en jeringa (caucho) pero muy rica en maderas nobles (Mesquita, 2012).

\section{El arte de la narración}

Las historias mitológicas entre los Asháninca se ocupan de los orígenes de la vida, pero representan y transmiten el conocimiento particular conceptualizado y las explicaciones de los procesos de la vida y de las trans- 
formaciones que crean las condiciones de vida y sus formas tal como se conocen. Observar las historias de los mitos ofrece la oportunidad de investigar y reconocer las formas de objetivación de la vida de una manera articulada, así como los principios en que se basan las diferentes formas de vida.

Las explicaciones mitológicas, al mismo tiempo, forman parte intrínseca de la percepción y las prácticas cotidianas del pueblo, en la medida en que postulan y afirman los principios básicos de la comprensión de los mitos. Ilustran los aspectos del mundo, generalmente invisibles e imperceptibles, y así explican los cambios, que también incluyen eventos históricos, y demuestran las consecuencias del comportamiento humano. En virtud de esto, con una perspectiva fenomenológica, busco lo que se verbaliza, lo percibido, lo sentido e internalizado a través de sus percepciones y experiencias. El significado de los mitos no sólo está representado en los actos verbalizados y símbolos de sonido. Una narración mítica revela su significado en su alcance, e incluye las acciones del narrador y la experiencia vivida por la audiencia. Se desarrolla no sólo en relación con su pasado u otros mitos, sino también en relación con el presente, el medio ambiente, el paisaje y los seres que lo habitan. Surge también en relación con los objetos manufacturados, y en relación con los rituales y las prácticas sociales.

Por lo tanto, el mito se inscribe en las prácticas, lugares, objetos, cuerpos, relaciones, producción, y finalmente emerge en la vida cotidiana de estas personas. Las acciones cotidianas traen posibilidades de contacto (consciente o inconsciente) más estrecho con seres no humanos cuando se cuenta un mito. Estas formas de representación, comunicación y textualidad sobrepasan las fronteras de lo oral y escrito, lo humano y lo no-humano y de lo social y lo natural. En este sentido, basado en la capacidad de inscribirse en otras formas textuales, el mito se convierte en -como Malinowski afirmó una vez- «no sólo una historia que se cuenta, sino una realidad vivida» (Malinowski, 1948 [1926], p. 100).

El "alma de un pueblo" o su Zeitgeist está en el vivir con y en relación con, es decir, en la experiencia con lo cotidiano. Así tenemos que las tradiciones no son necesariamente transmitidas sólo por vía oral, como se mencionó antes. Por lo tanto, hago hincapié en que 'las tradiciones orales o no' no constituyen un corpus estático, listo para ser capturado y registrado. Más bien, son siempre nuevas las versiones narrativas y discursos producidos en circunstancias y en función de los actores de la comunicación (Bakhtin, 2006, p. 116). 


\section{Metodologia}

Originariamente desarrollada en el estudio de sociedades de pequeña escala, la etnografía tiene como fundamento el contacto, confrontación, diálogo con el otro. Nuestro objetivo ha sido la búsqueda de comprensión de los mitos, historias y vivencias acerca del consumo de su bebida ritual: el Kamarampi. La elección de un camino para nuestras investigaciones no ocurre al azar, pero depende del objeto, del enfoque o de cómo deseamos abordarlo. Hemos llevado a cabo esta investigación en conjunto con la etnografía en nuestro trabajo de campo con los Asháninca, hemos vivido con ellos durante unos ocho meses, comiendo, tomando baños en el río, jugando con los niños, recogiendo papas con las mujeres, la pesca en las playas en el verano, hablando con los mayores, con los sheripiari, buscando vivir como uno de ellos. Por lo tanto, dejarse envolver por esta perspectiva no es perder el rumbo, sino abrirse para nuevas posibilidades de conocimiento del nuestro objeto.

\section{El Sheripiari ${ }^{4}$ o Chamán}

Para los Asháninca, todas las cosas vienen de un mismo espíritu y son parte de este, incluso ellos mismos. En este conjunto sistémico, los Kampa también se introducen en relación con otros seres. Veneran los fenómenos de la naturaleza y no solo creen en la potencia de los recursos que permiten la vida, sino también en el rigor de los dioses. Para mediar en la ambivalencia entre el bien y el mal, está el chamán o sheripiari, que tiene como tarea y compromiso con su pueblo mirar el plano invisible. El papel del chamán como mediador entre lo visible y lo invisible es característica de los sistemas chamánicos de los pueblos indígenas que habitan en el vasto territorio de la selva amazónica (Renard-Casevitiz, 1998; Weiss, 1969; Santos, 2009). El sheripiari es quien invoca el poder o los favores de los dueños de la fauna, la flora y los fenómenos meteorológicos para lograr una buena siembra, la presencia de los alimentos y la abundancia de la cosecha. Es, también, quien invoca la fuerza de los "dueños de los fenómenos meteorológicos", como Inkaniteri "dueño de la lluvia" y Pawa, que representa el Sol.

Los sheripiari son titulares de la negociación entre los distintos mundos que conforman su cultura y responsables de llevar a cabo acuerdos con los "dueños" de la selva, animales, ríos o agua, por lo que ciertas reglas deben cumplirse. El cumplimiento de estas reglas garantiza a los Asháninca el éxito en la agricultura y en la caza, lo cual redunda en una buena alimentación y ausencia de enfermedades. El "dueño de los animales" es 'Nantatsiri' y el de las plantaciones es Oretsi, que permite a los Asháninca tener una cosecha buena o mala. Noonki 
es el "dueño de las aguas y de los seres de las aguas", y sólo el sheripiari es capaz de negociar y mediar la relación entre los Asháninca y los seres de este mundo. A la luz de los datos recogidos en mi trabajo de campo junto a los Asháninca, concuerdo con Viveiros de Castro (2004, pp. 228-229) en que los "dueños de la plantación" y "los dueños de los animales" son

(...) espíritus-maestros invariablemente dotados de una intención similar a la humana, como hipóstasis de la especie animal a la que se asocian, creando un campo intersubjetivo animal-humano donde los animales empíricos no están espiritualizados. Obsérvese que la distinción entre animales vistos en su apariencia-alma y los espíritus-maestros de la especie no es siempre clara o pertinente; por otra parte, siempre es posible toparnos en el bosque con algo que parece ser simplemente un animal, pero que resulta ser el disfraz de un espíritu de naturaleza completamente distinta.

(Traducción de la autora)

Por lo tanto, es necesaria la intermediación del chamán para comunicarse con los dueños de las cosas y acordar los intercambios. El mundo Asháninca es marcado por dualismos que oscilan entre el bien y el mal. Existe una relación entre el bosque y la tierra, porque para ellos, todo lo que existe en la naturaleza es conectado, como un gran sistema que integra la cosmología Kampa. Los mundos y espacios míticos y la naturaleza están estrechamente vinculados y tienen en la Lupuna (pasawoteya-tsomonto) y el bosque (ãtami) espacios míticos que sirven de conexión entre los mundos.

El bosque es un espacio mítico sagrado porque fue allí donde vivió Pawa cuando estuvo presente en la Tierra y donde los espíritus fuertes también habitan. Para los Asháninca, el bosque emana una gran energía y se observa el sol, la luna y las estrellas con mayor claridad. De ahí la importancia de los bosques para la Tierra y el Universo como un todo. El bosque también está marcado por la ambigüedad: a veces se percibe como donante de recursos para la supervivencia indígena y lugar sagrado, y otras veces se ve como la morada de los espíritus malignos como el "peyiari" (almas, espíritus malignos), que deambulan por el bosque.

A lo largo de la vida de un sheripiari, los espíritus que habitan en los diversos mundos le acompañarán y enseñarán acerca de todas las cosas. Él puede dominar estos seres mágicos, porque siempre está alerta. Él habla con el Kapironkari (dueño de las plantas llamadas bambú), con Noonki (propietario de las 
aguas y la vida acuática), con Nantatsiri (dueño de los animales). Es capaz de negociar sobre la caza y la pesca en esta mediación entre los mundos para que la gente que esté bajo su protección tenga alimentación. Él hace convenios con Oretsi (dueño de las plantaciones) para que pueda permitir una buena cosecha y evitar los males.

\section{El Kamarãpi (Ayahuasca) y algunas narrativas}

Es a través del lenguaje verbal que los Asháninca expresan sus experiencias diarias con el fin de entretener y/o transmitir información a su gente. A través de dibujos, relaciones, percepciones y una serie de conocimientos nativos hay una gran acumulación de saberes que se han logrado con el tiempo y su proceso histórico, llegando hasta la actualidad. La exposición de la potencia de su conocimiento, la creación de un espacio para la realización del potencial de la alteridad y de ir más allá de los límites humanos, son lo que nos proponemos analizar en dos formas de transmisión del conocimiento Asháninca.

La primera es lo que se narra a través de dibujos. El conocimiento Asháninca está más allá de las narrativas; está en sus trajes, en sus artesanías y en su vida cotidiana. En la figura siguiente, tenemos la representación de Maankekõta (camino de la serpiente), que es una serpiente muy presente en las áreas de la selva tropical del Amazonas habitada por Asháninca. Esta serpiente es emblemática porque todos la tienen miedo y la veneran debido a su fuerza y potencia en todos los mundos. De acuerdo con los Asháninca, esto se debe a que las representaciones son también reconocidas por las deidades. La serpiente es considerada un ser encantado y ellos utilizan sus representaciones simbólicas y contradictorias en su día-a-día en la medida en que estas representaciones contribuyen a difundir y salvaguardar la memoria colectiva Asháninca ${ }^{5}$.

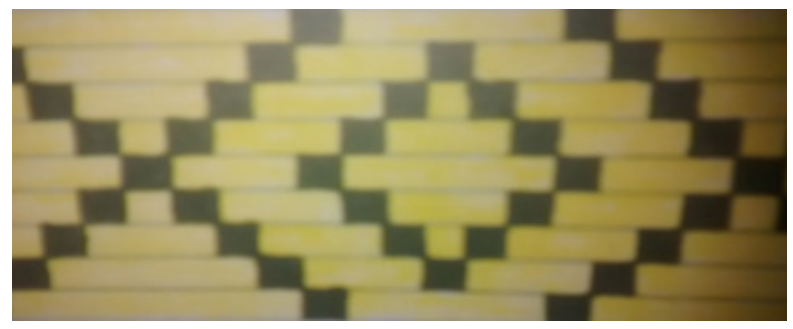

Fig. 1. Representación de una serpiente (foto de la autora, 2013) 
La segunda es la narración de un mito Asháninca contado por un chamán en un momento después de que él hizo uso de sus plantas maestras como Ishiko, Koka y Chamairo.

(...) Yo estaba a caminar por el bosque y a cazar tsimeri, yo estaba sólo y empezaba la noche. Había mambeado Koka, y los espíritus del bosque estaban enojados, podía sentir, pero fui a buscar comida. Yo quería un pollo perdiz. Yo miraba por el camino, escuchaba uno silbido, pero no miraba nada. La noche cayó y nada de una caza. Estaba a caminar por el bosque cuando sentí un golpeo en mi espalda, miré y no pude mirar nada. Estaba tranquilo y desde ese momento sabía que no estaba solo, y que estaba a recibir lecciones y me quedé por allí toda la noche. Casi cuando llegó el día claro y no logré ninguna caza. Triste venía en dirección a mi casa y casi cerca, muy cercano mismo, maté a un pavón. Llegué en mi casa con comida. (...) Comprendí lo los espíritus estaban a enseñar a mí en el bosque, y estoy contento.

(Cuaderno de campo de la autora, 2014)

En este relato, el chamán transmite sus conocimientos y su experiencia a sus familiares. Los conocimientos experimentados y vividos de una manera completa por un chamán no sólo son importantes escuchar, sino aprender por la experiencia. Por lo tanto, este conocimiento es escuchado por sus familiares, pero muchos buscan vivir y por lo tanto dejan que ese conocimiento esté en sus mentes y en sus cuerpos; esta es la forma en que aprehenden su mundo de la vida.

Algunas representaciones de la cosmología Asháninca traen a la luz el conocimiento incorporado en la memoria colectiva y serán -muchos de ellos- con sus experiencias y reinterpretaciones, presentados con un proceso pulsante en sus historias, narrativas y cosmologías. Proceso que además es hermoso y rico, y que se reincorpora a la experiencia de cada indígena. Por lo tanto, teniendo en cuenta que la acción de narrar o contar permite a todas las sociedades de hace algunos miles de años, señalar la importancia de la narrativa en la difusión de los conocimientos, creencias y valores de muchas sociedades donde hay un predominio de la tradición de cada pueblo.

Es importante tener en cuenta cómo estas narrativas revelan acerca de los conocimientos tradicionales, creencias y valores de los Asháninca. Así, teniendo en cuenta que todavía con la inclusión de estas personas en el mundo blanco, esta tradición durará por muchas años en sus diferentes campos sociales que están en constante proceso de reafirmación de su cultura, así como convertirse en una manera de vivenciar los cambios constantes que están experimentando. 


\section{Las narrativas Asháninca}

Los principales argumentos de Claude Lévi-Strauss $(1988,1989)$ se puede empezar a pensar en la variabilidad de las formas narrativas de los Asháninca. Partiendo de una crítica de la perspectiva clásica y criticando, en particular la noción de Lévy-Bruhl (2010 [1935]: p. 33), de la mentalidad primitiva, él creó el concepto de pensamiento salvaje, muy cerca del pensamiento mítico, redactado por Maurice Leenhardt (1971).

Esta no es la idea de la naturaleza, basándose únicamente en la comunicación oral, pero el diseño de la actividad espontánea del espíritu humano, un pensamiento altamente simbólico que revela la diversidad de la cultura humana, que apunta a la existencia de movimientos regulares universales en el funcionamiento del espíritu humano. Por lo tanto, entendemos la definición de mito como una creación narrativa simbólica atemporal y universal, ya que, según este punto de vista, es una de las mayores expresiones de este espíritu humano (Lévi-Strauss, 1989, pp. 152-205). La entrada de las cuestiones de historicidad y los diferentes marcos de tiempo, como el tema del cambio cultural después del contacto con las sociedades colonizadas, se acompaña de una multitud de estudios que integran la dimensión diacrónica.

En esta perspectiva, el lenguaje es uno de los elementos más importantes que afirman el carácter social de la memoria como los intercambios entre los miembros de un grupo se realizan a través de ese medio. En otras palabras, el lenguaje es el instrumento de socialización de la memoria, ya que reduce, tonifica y aporta en el mismo espacio histórico y cultural, las experiencias tan diversas como el sueño, los recuerdos y los experimentos recientes (Halwacks, 1990: 183). Las representaciones de las cosmologías de los Asháninca en sus diversas historias se presentan como su forma de relacionarse con el mundo, tanto de su mundo, como en el mundo de los wiracocha (blancos).

Por lo tanto, el trabajo con las narrativas de un pueblo indígena se realiza mediante la transferencia de recuerdos que se dan por la tradición oral y a través también de dibujos. El acto de narrar, de acuerdo con Abramovich (1987, p. 22), es enseñar lo otro para escuchar, pensar y "ver con los ojos de la imaginación". Por lo tanto, es importante el papel de las narrativas a través de sueños y visiones de Kamarampi, así como sus expresiones, hechos por los sabios Asháninca. 
De acuerdo con Métraux (1973, p. 54), «el poder de cual estos hombres son portadores - por su capacidad de comunicarse con los espíritus-proviene de su acceso a un saber oculto a los otros hombres, en teoría, otorgado a través de los sueños». Para los Asháninca, los sueños son también una forma de acceso a otro mundo, el mundo de los espíritus, refiriéndonos a la concepción del mundo de los cielos, la tierra y el inframundo. Amixiuiere (entre estar despierto y estar dormido) es un momento importante para que el chamán también tenga acceso a otros mundos y cuerpos. Santos (2009, p. 182) señala que los sueños tienen el poder de predecir eventos y son una forma de vida para la reflexión, e indica que

(...) ashimajeita es una forma que el hombre descansa en la cama y luego duerme y el pensamiento interior inicia la actividad de 'representar la realidad en la fantasía' mientras uno está dormido, como sucesos o imágenes de película, a lo que llamamos como 'personificación del sueño’ cuando uno está dormido. Es una cosa que uno piensa acerca de realidad o fundamento y, en especial, proyecto, deseo, esperanza que en el período que está descansando lo vive y al despertar uno recordamos con la probabilidad de realizarse en un período cercano. Para el Asháninka, el sueño es la falta de atención o ser demasiado negligente en la vida real. Pero los sueños te vaticinan el presente que se viene o va a suceder de un día a tres en delante de lo que uno soñó. Por ello, el Asháninca confía los anuncios de acciones que va ocurrir en el futuro-presente en sus sueños y lo relaciona con sus diferentes actividades diarias en pesca, caza y otras acciones que realiza.

Con relación al Sheripiari, observa Varese:

El chamán cura, vende a la muerte o la puede enviar contra los enemigos. El chamán es el héroe que ha superado todo los obstáculos y las pruebas en su camino iniciático; ha logrado ser el esposo del tabaco, sheri que es mujer u le facilita el vuelo mágico, ha logrado dominar otras plantas alucinógenas, puede transformarse en pájaro, viajar al mundo de los muertos o repetir sin dificultad las acciones que las divinidades realizaron en el principio de los tiempos.

(Varese, 1968, p.132). 
La experiencia y la representación del cosmos se encuentran en el legado de acciones de los seres sobrenaturales en la cultura Asháninca. También a través de las historias contadas y vividas en el tiempo mítico o no, establecen el vínculo entre el hombre y las más diversas realidades y mundos. En la perspectiva Asháninca, el momento actual que están experimentando se caracteriza por la desestabilización del cosmos, que amenaza la supervivencia de la vida de ellos en los bosques y así en la tierra. Estas desestabilizaciones en el equilibrio, esta forma tan radical, tienen que ver con el destino causado por el hombre a sí mismo. Y Sheripiari que es un maestro pero al mismo tiempo un estudiante de Kamarampi (Ayahuasca) ${ }^{6}$ tiene la traducción de los matices de su mundo en este planeta.

Por lo tanto, el sheripiari es también un gran narrador de historias, el que experimenta la historia de una forma más ardiente, sugestivamente, que narra de forma natural, que sabe la trama y domina su público. Es el titular de la memoria colectiva, la cultura y la tradición de su pueblo. Él sabe cómo usar la palabra y en especial frente a su público participa utilizando el tiempo, el espacio y el encantamiento para mantener la atención. Al contar la historia, se socializa, recrea, forma, informa, educa, enriquece el lenguaje, estimula la imaginación y la inteligencia, despierta emociones, desarrolla el pensamiento lógico, pensamiento crítico y enseña a escuchar y a vivir como un Asháninca.

\section{Conclusiones}

En este artículo presentamos narraciones y mitos que los Asháninca brasileños nos transmiten sobre algunas de sus cosmovisiones. Aunque no establecemos concluciones definitivas, sí brindamos observaciones acerca del mundo en que los Asháninca viven y de la importancia que posee el Kamarampi para la comprensión de los procesos e interconexiones entre este mundo y los otros que forman parte de su cosmología. Por supuesto, es notoria la manera de expresión de los mitos, historias y experiencias vividas y que van, además de la palabra dicha, representadas por las pinturas corporales, dibujos en cushmas y en sus artesanías. Los Asháninca se comunican de muchas maneras como en las que los iniciados detienen el dominio, o sea, la interlocución con la naturaleza, animales, bosques y plantaciones, y esa comunicación sigue sucediendo en su día a día, transcurren generaciones y se constituye también en el modo de ser Asháninca, que los hacen únicos. 


\section{Referencias bibliográficas}

Leenhardt, M. (1971). Do Kamo, la personne et le mythe dans le monde mélanésien. Gallimard, Paris.

Lévi-Strauss, C. (1978;1989). Mito e Significado. Edições 70, Lisboa.

Lévi-Strauss, C. $(1970 ; 1983 ; 1988 ; 1989)$. O Pensamento Selvagem. Papirus Editora/ Companhia Editora Nacional, Campinas/ São Paulo.

Metraux, A. (1973). Religión y magias indígenas de América del Sur. Ed. Aguilar, Madrid.

Mesquita, E. (2012). Ver de perto pra contar de certo: as mudanças climáticas sob os olhares dos moradores da floresta do Alto do Juruá. Tese doutorado, mimeo, UNICAMP, Campinas.

Pollock, D. (1992). Culina shamanism: gender, power and knowledge. In: E. Jean Langdon \& G. Bear (orgs.). Portals of Power: shamanism in South America. Albuquerque: University of New Mexico Press. pp. 25-40, USA.

Renard-Casevitz, F. M. (1998). Ideologia Matsiguenga: entre lo local e lo Global, Año XVII, n 17, PUCP, Lima.

Romani, M. M. (2004). La toponímia en el Gran Pajonal con especial atención a los topónimos de filiación asháninka. Universidad San Marcos. Dissertación in Linguística. mimeo, Lima.

Jacintos Santos, P. E. (2009). Expresiones orales iconográficas en el guerrero arawak-asháninka. Perú, USMA, Lima.

Varese, S. (1968). La sal de los cerros. Notas etnográficas e historia sobre los campos de la selva del Perú. Lima. Universidad Peruana de Ciencias y Tecnología. Lima.

Viveiros De Castro, E. (2004). Perspectivismo e multinaturalismo na América Indígena, o que nos faz pensar; n 18, setembro, São Paulo.

Zolezzi, E. R. (1986). Los Ashaninka, un pueblo tras el bosque. Contribución a la etnología de los Campa de la Selva Central. Ed. PUCP. Lima. 
Zolezzi E. R. (1996). Los ciclos de Pachakama, Inka y Sacramento en la mitología campa-asháninka como interpretaciones de los procesos de reemplazo tecnológico y subordinación económica surgidos de la colonización. In: Anthropológica. PUCP; Vol. 11; pp.109-154, Lima.

Zolezzi, E. R. (1997). Los Asháninkas: Un pueblo tras el bosque. Contribución a la etnología de los campos de la selva central. Universidad Católica del Perú, Lima.

Zolezzi, E. R. (1999). Origen y clasificación de las plantas cultivadas en el pensamiento mítico asháninka. Antropológica ,15. pp. 255-288. Lima.

Weiss, G. (1969). Campa Cosmology. The world of a forest tribe in South America. Anthropological Papers of the American Museum of Natural History. New York.

${ }^{1}$ Los Asháninca también recorrían las cabeceras del río Envira, donde en la actualidad hay también una reserva que pertenece a ellos.

${ }^{2}$ Según Romaní (2004) "algunas variedades lingüísticas que presenta la lengua Asháninca se reducen a diferencias léxicas y fonéticas," y por lo tanto pueden distinguir las diferencias por región, como Ashaninka do Rio Tambo e Ene, Ashéninka do Pichis, Ashaninka de Pajonal, Ashaninka de Ucayali e Yuruá, Ashaninka de alto Perené y Ashaninka Apurucayali.

${ }^{3}$ Actualmente Yanesha.

${ }^{4}$ Es en el sentido del Zolezzi (1986) el significado de la palabra sheripiari es una unión de dos -Términos - peyari 'espíritu' y Sheri-'tabaco '- que en conjunto significa' espíritu del tabaco. Para el autor, peyari sólo serían una transformación del fonema. Eso no es lo que encontramos como la comprensión de peyari entre nuestros interlocutores, como se ve en el texto.

${ }^{5}$ Esos dados fueran colectados en mi pesquisa de campo con los Asháninca brasileños.

${ }^{6}$ Una de las herramientas que pueden facilitar el acceso de los chamanes estas áreas es Ayahuasca, que es una cocción de plantas sagradas, la cual se prepara el té de vides Banisteriopsis caapi y otras plantas que se mezclan, por ejemplo, Psychotria viridis y Diplopteryscabrerana a través de la miles de años, y se ha utilizado durante más de cuarenta grupos indígenas, principalmente en la cuenca del Amazonas y del Orinoco, para la adivinación, curación y otras razones cosmológicas, además de expresar sus percepciones del mundo. 